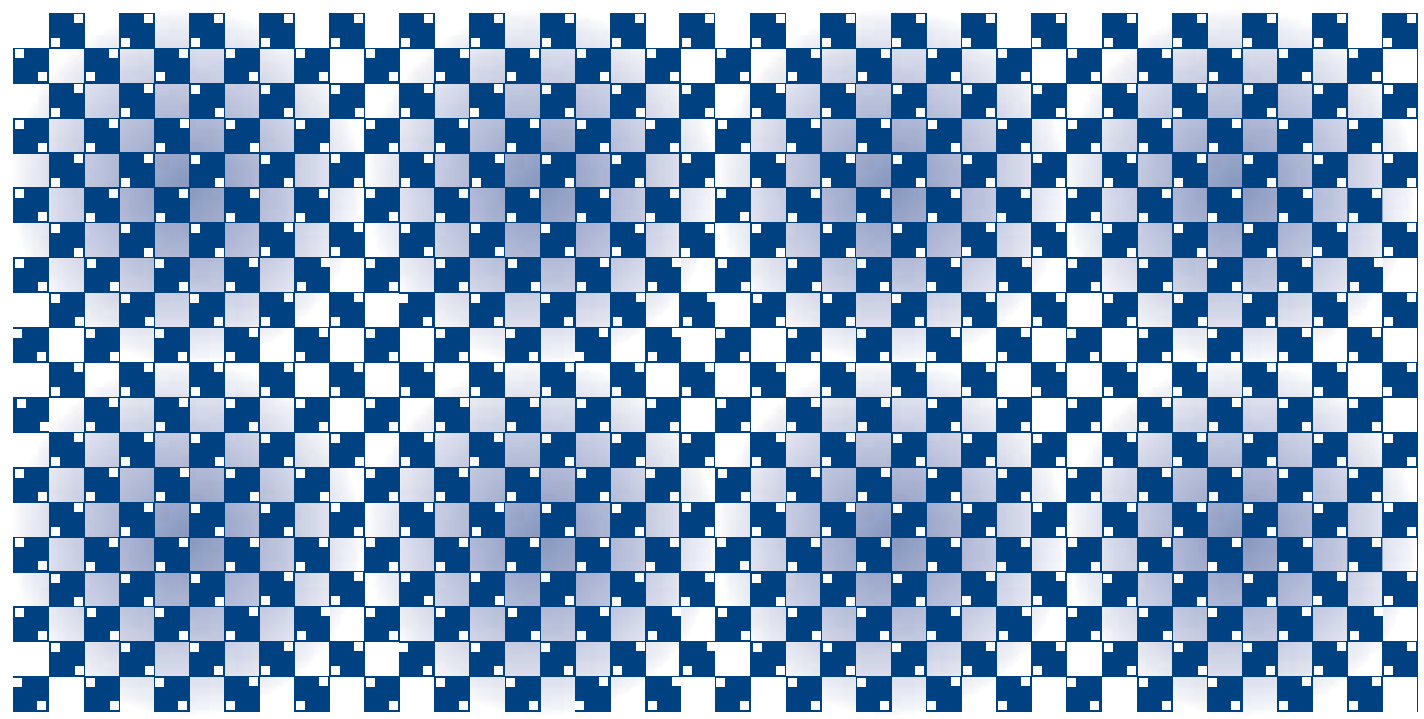

DEVELOPMENT

\title{
Comfortably Numb
}

Numb, an important regulator of asymmetric cell division and cell-fate determination, has been implicated in different aspects of neural development in mammals. But the traditional approach of eliminating the expression of a gene to investigate its functional role has not been particularly informative in the case of $N u m b$, as knockout mice die early in embryogenesis. Two research groups have now circumvented this problem by generating conditional Numbknockout mice in which expression is selectively abolished in restricted areas of the neural tube.

Klein et al. ablated the Numb gene in neuroepithelial cells of the midbrain-hindbrain boundary using the Cre-lox system. Specifically, they crossed mice in which Numb was flanked by loxP sequences with mice in which Cre recombinase - an enzyme necessary for recombination at loxP sites - was under the transcriptional control of the engrailed-2 promoter, which is characteristic of the midbrain-hindbrain boundary. After recombination, Numb was absent only cells in which engrailed-2 was active.

The authors focused their attention on the development of the cerebellum, which originates from the midbrain-hindbrain boundary. They found that the absence of Numb in the mutant mice led to a delay in the maturation of mitotic precursors into granule cells and in the subsequent migration of this cell type into the granule cell layer, but had no effect on the proliferation of precursors. In addition, the elimination of $N u m b$ resulted in a delay in the maturation and layered organization of Purkinje cells, which might be a consequence of both the abnormal development of granule cells and the absence of Numb itself. Ultimately, these defects led to macroscopic cerebellar defects such as abnormal foliation and reduced size.

In the second study, Li et al. used a similar system for the generation of conditional knockouts, but focused on the dorsal forebrain by using the Emx1 promoter. These authors were also interested in the possibility of redundant functions of Numb and its homologue Numblike. They therefore generated the conditional knockout mice in a Numblike-null background. In addition, the use of the Emx1 promoter, which is active from embryonic day (E) 9.5, enabled them to study the role of Numb in the second wave of cortical neurogenesis, which occurs from E12.5, while sparing the first wave, which starts from E8.5. Li et al. found increased proliferation and apoptosis of neural precursors in the double-mutant animals - phenotypes that were accompanied by a reduction in the differentiation of these progenitors into neurons and by abnormalities in the morphogenesis of the cortex.

Despite the clear differences in genetic background between the animals that were used in both studies, the data disclose a critical role for Numb in the differentiation of mitotic precursors into mature neurons in two brain regions. However, the underlying mechanisms might be markedly different, as indicated by the differential effect of the mutation on precursor proliferation in the cortex and the cerebellum. Considering the large number of potential binding partners that have been described for Numb, there is scope for multiple downstream signalling mechanisms that might mediate its action, but unravelling them will constitute a considerable experimental challenge.

Juan Carlos López

(2) References and links ORIGINAL RESEARCH PAPERS Klein, A.-L. et al. Murine Numb regulates granule cell maturation in the cerebellum. Dev. Biol. 4 December 2003 (doi: 10.1016/j.ydbio.2003.10.017) | Li, H.-S. et al. Inactivation of Numb and Numblike in embryonic dorsal forebrain impairs neurogenesis and disrupts cortical morphogenesis. Neuron 40, 1105-1118 (2003)

FURTHER READING Jan, Y.-N. \& Jan, L. Y. Asymmetric cell division in the Drosophila nervous system. Nature Rev. Neurosci. 2 , 772-779 (2001)

\section{IN THE NEWS}

Atkins diet proposed for epilepsy

Never out of the news for long, the Atkins diet hit the headlines again recently when a group of US scientists reported that the low-carbohydrate, high-protein diet could help to control epilepsy in children.

According to $B B C$ Online (10 December 2003), "Researchers from the Johns Hopkins University in Baltimore, Maryland, studied six patients, including three patients who were aged 12 or younger." After following the Atkins diet, "Two children and one young adult were completely free of seizures, and were able to cut down on their use of anti-convulsant medications. The patients stayed on the diet, and free of seizures, for as long as $\mathbf{2 0}$ months."

The finding that the controversial diet can restrict seizures is not that surprising, given that that many children with epilepsy already control their seizures by following a more restrictive lowcarb, high-fat diet. Such 'ketogenic' diets, like the Atkins diet, produce ketones as a byproduct of fat metabolism, and the ketones are thought to inhibit seizures.

However, the diet that was used in the new study was considerably less restrictive than the conventional ketogenic diet. According to one of the authors, Eric Kossof, "the report raises new questions about the ideal level of calorie and protein restriction imposed by ketogenic diet," (The Guardian, 10 December 2003). He hopes that "by learning more about how the Atkins diet works to control seizures, we should learn more about which patients may benefit best from either of these diets."

Rachel Jones 